\title{
Carotenoids but not flavonoids are associated with improvements in spatial working memory in younger adults in a flavonoid-rich v. -poor fruit and vegetable intervention study
}

\author{
A. L. Macready ${ }^{1,2}$, L. T. Butler ${ }^{2}$, O. B. Kennedy ${ }^{1}$, T. W. George ${ }^{1}$, M. F. Chong $^{1}$, \\ J. A. Lovegrove ${ }^{1}$ and the Flavurs team ${ }^{1} \dagger$ \\ ${ }^{1}$ Hugh Sinclair Human Nutrition Unit, Department of Food and Nutritional Sciences, University of Reading, Whiteknights, \\ Reading RG6 6AP and ${ }^{2}$ School of Psychology and Clinical Language Sciences, University of Reading, Whiteknights, \\ Reading RG6 6AH, UK
}

Findings from animal studies suggest that components of fruit and vegetables (F\&V) may protect against, and even reverse, age-related decline $^{(1,2)}$ in aspects of cognitive functioning such as spatial working memory (SWM). Human subjects in vivo and in vitro studies indicate that anti-inflammatory, anti-oxidant and cell-signalling properties of flavonoids and carotenoids, non-nutrient components of $\mathrm{F} \& \mathrm{~V}$, may underpin this protective effect ${ }^{(3-5)}$. The Flavonoid University of Reading Study (FLAVURS), designed to explore the doseresponse relationship between dietary $F \& V$ flavonoids and CVD, enabled the investigation of such an association with SWM.

FLAVURS is an 18-week parallel three-arm randomised controlled dietary intervention trial with four time points, measured at 6-weekly intervals from baseline. Low F\&V consumers at risk of CVD aged 26-70 years were randomly assigned to high flavonoid (HF), low flavonoid (LF) or control group. F\&V intake increased by two daily $80 \mathrm{~g}$ portions every 6 weeks, with either HF or LF F\&V, in addition to each participant's habitual diet, while controls maintained their habitual diet. At each visit, participants completed a cognitive test battery with SWM as the primary outcome.

The HF group showed significantly higher levels of urinary flavonoids than LF or controls at 12 weeks $(P<0.001)$ as expected, but surprisingly only higher levels than LF at 18 weeks $(P<0.01)$. The LF group showed higher levels of plasma carotenoids than the other groups at 18 weeks $(P<0.001)$. No group differences were found for SWM overall, however, age-group sub-analyses $(26-50$ and 51-70 years of age) showed differences from 0 to 18 weeks for younger adults, with LF improving significantly more than the other two groups on SWM $(P<0.05)$. As nutritional absorption is known to decrease with age, separate stepwise regressions were performed on the two age groups irrespective of dietary group, with urinary flavonoids and plasma carotenoids as predictors. For younger adults, improved SWM performance from 0 to 18 weeks was associated with higher carotenoid levels, $\beta=0.28, t(55)=2.10, P<0.05$, accounting for $7.5 \%$ of the variance, $R^{2}=0.075, F(1,54)=4.41, P=0.040$. For older adults, no between-group SWM differences were found.

\begin{tabular}{|c|c|c|c|c|c|c|}
\hline \multirow[b]{2}{*}{ Dietary group } & \multicolumn{3}{|c|}{ Younger adults (26-50 years of age) } & \multicolumn{3}{|c|}{ Older adults (51-70 years of age) } \\
\hline & $\mathrm{N}$ & Mean & SE & $\mathrm{N}$ & Mean & $\mathrm{SE}$ \\
\hline High flavonoid diet & 30 & -0.17 & 0.16 & 27 & -0.05 & 0.16 \\
\hline Low flavonoid diet & 28 & $0.53^{*}$ & 0.21 & 31 & 0.06 & 0.20 \\
\hline Habitual diet & 21 & -0.24 & 0.22 & 35 & 0.28 & 0.15 \\
\hline
\end{tabular}

Mean change in SWM performance over time (0-18 weeks), with higher scores indicating improved performance. Mean natural log transformed $z$-scores are significantly improved for LF compared with HF and HD groups (ANOVA, followed by Dunnett's test): $* P<0.05$

Findings suggest that F\&V-based flavonoids and carotenoids may provide benefits for cognitive function, and that carotenoids in particular may improve cognitive performance in SWM. Given that these benefits were restricted to younger adults, future work is needed to test the reliability of this finding, as well as determine the mechanisms by which age-dependent differences in F\&V responsiveness occur.

1. Joseph JA, Shukitt-Hale B, Denisova NA et al. (1999) J Neurosci 19, 8114-8121.

2. Williams CM, El Mohsen MA, Vauzour D et al. (2008) Free Radic Biol Med 45, 295-305.

3. Spencer JPE (2007) Genes Nutr 2, 257-273.

4. Letenneur L, Proust-Lima C, Le Gouge A et al. (2007) Am J Epidemiol 165, 1364-1371.

5. Akbaraly NT, Faure H, Gourlet V et al. (2007) J Gerontol A Biol Sci Med Sci 62, 308-316. 